\title{
Treatment-failure tularemia in children
}

\author{
Arzu Karlı, MD', Gülnar Şensoy, MD', Şule Paksu, MD², Muhammet Furkan Korkmaz, MD², Ömer Ertuğrul, MD², Rifat Karlı, MD³ \\ ${ }^{1}$ Division of Pediatric Infectious Diseases, Ondokuz Mayis University Faculty of Medicine, Samsun, ${ }^{2}$ Department of Pediatrics, Ondokuz Mayis University Faculty of \\ Medicine, Samsun, ${ }^{3}$ Departments of Otorhinolaryngology, Ondokuz Mayis University Faculty of Medicine, Samsun, Turkey
}

Purpose: Tularemia is an infection caused by Francisella tularensis. Its diagnosis and treatment may be difficult in many cases. The aim of this study was to evaluate treatment modalities for pediatric tularemia patients who do not respond to medical treatment.

Methods: A single-center, retrospective study was performed. A total of 19 children with oropharyngeal tularemia were included.

Results: Before diagnosis, the duration of symptoms in patients was $32.15 \pm 17.8$ days. The most common lymph node localization was the cervical chain. All patients received medical treatment (e.g., streptomycin, gentamicin, ciprofloxacin, and doxycycline). Patients who had been given streptomycin, gentamicin, or doxycycline as initial therapy for 10-14 days showed no response to treatment, and recovery was only achieved after administration of oral ciprofloxacin. Response to treatment was delayed in 5 patients who had been given ciprofloxacin as initial therapy. Surgical incision and drainage were performed in 9 patients $(47.5 \%)$ who were unresponsive to medical treatment and were experiencing abcess formation and suppuration. Five patients (26.3\%) underwent total mass excision, and 2 patients (10.5\%) underwent fine-needle aspiration to reach a conclusive differential diagnosis and inform treatment.

Conclusion: The causes of treatment failure in tularemia include delay in effective treatment and the development of suppurating lymph nodes.

Key words: Tularemia, Treatment failure, Surgical procedures

\section{Introduction}

Tularemia is a zoonosis caused by a facultative intracellular gram-negative coccobacillus, Francisella tularensis. ${ }^{1)}$ A large number of tularemia outbreaks in European countries, including Turkey, have been found to be linked to the ingestion of contaminated food or water. Almost all tularemia cases are presented with oropharyngeal tularemia and neck mass in this region. The most common clinical signs are sore throat, fever, swelling on the neck, skin, mucosa, or conjunctival inoculation lesions. ${ }^{2-4)}$

The diagnosis of tularemia mainly relies on clinical suspicion. The isolation of $F$. tularensis in sterile body samples is the gold standard in the diagnosis. Because this method is very difficult and risky to laboratory staff, diagnosis of tularemia depends on clinical signs and serological findings. ${ }^{5,6)}$

Early diagnosis, and initiating effective antibiotic treatment, are still the corner stones of successful therapies. In many cases, effective treatment success rates are still very high, although rare cases are treatment failure. ${ }^{7-9)}$ In this article, we evaluated clinical features and treatment modalities for treatment failure cases among our pediatric tularemia patients.
Corresponding author: Muhammet Furkan Korkmaz, MD

Deparment of Pediatrics, Ondokuz Mayis University, Faculty of Medicine, Kurupelit, Samsun, Turkey

Tel: +90-362-312-1919-4040

Fax: +90-362-4576041

E-mail: korkmazmfurkan@gmail.com

Received: 11 July, 2017

Revised: 10 September, 2017

Accepted: 15 December, 2017
Copyright ( $\odot 2018$ by The Korean Pediatric Society

This is an open-access article distributed under the terms of the Creative Commons Attribution NonCommercial License (http://creativecommons.org/ licenses/by-nc/4.0/n which permits unrestricted noncommercial use, distribution, and reproduction in any medium, provided the original work is properly cited. 


\section{Materials and methods}

The study was carried out as a single-center and retrospective study among 19 pediatric oropharyngeal tularemia patients with no response to medical treatment, who were reviewed retrospectively from December of 2009 through April of 2012 at the pediatric infectious disease department of the Ondokuz Mayis University Hospital, Samsun, Turkey (a tertiary care hospital with a total of 1,000 beds, including 200 for pediatric patients).

In this study, tularemia was defined as the presence of compatible clinical signs or symptoms, together with the presence of specific antibodies at significantly higher titres $(\geq 1: 160)$ in microagglutination testing (MAT). The tularemia MAT was performed at the Public Health Institution of Turkey (In the literature, the sensitivity and specificity of MAT were $97.6 \%$ and $98.7 \%$, respectively. $\left.{ }^{8}\right)$. The tularemia patients who presented neck mass with or without fever had received antimicrobial treatment at another hospital before being referred to our hospital.

Streptomycin (30 mg/kg/day intramuscularly, 2 times daily), ciprofloxacin ( $20 \mathrm{mg} / \mathrm{kg} / \mathrm{day}$, per orally, 2 times daily), gentamicin $(7.5 \mathrm{mg} / \mathrm{kg} /$ day intravenously, 3 times daily), and doxycyline (2$4 \mathrm{mg} / \mathrm{kg} /$ day, per orally, 2 times daily) were used in treatment, according to the patients' age.

Therapeutic failure was defined as the presence of one of the following: increase in size or appearance of new, enlarged lymph nodes; persistent or recurrent fever; constantly elevated or increasing erythrocyte sedimentation rate (ESR) and/or C-reactive protein (CRP) levels; or suppurated lymph nodes despite effective medical treatment (10-14 days).

Statistical analyses were performed by using SPSS ver. 15.0 (SPSS Inc., Chicago, IL, USA). The characteristic data are presented as number (\%) for categorical variables, and as mean \pm standard deviation or median (interquartile range) for continuous variables, as appropriate. This study was approved by the Institutional Review Board of Ondokuz Mayis University Faculty of Medicine (20120123), and the requirement for informed patient consent was waived.

\section{Results}

A total of 19 pediatric patients (10 female, 9 male patients) with tularemia were included in this study. Mean patient ages were 10.5

Table 1. Assessment of neck lymph nodes

\begin{tabular}{lc}
\hline Lymphadenopathy & Number \\
\hline Submandibular lymph nodes involvement & 8 (right, 7; bilateral, 1) \\
Cervical chain in different locations involvement & 15 (right, 7; left, 5; bilateral, 3) \\
Parotid/preauricular lymph nodes involvement & 0 \\
Post auricular involvement & 1 \\
\hline
\end{tabular}

years (range, 3.5-17 years). Most patient (68.4\%) lived in rural areas, $31.5 \%$ patient lived city area. Among the patients, $73.6 \%$ had a history of consumption of spring water and $26.3 \%$ patients contact with rodents. Before the diagnosis, the duration of symptoms in patients $32.15 \pm 17.8$ days. All patients were swelling on the neck, in addition 31.5\% patient were fever. The most common localization of the enlarged lymph node was detected at the cervical lymph node group (Table 1). Lymph node sizes were measured by ultrasound as minimum $2 \times 1 \mathrm{~cm}$ and maximum $8 \times 3 \mathrm{~cm}$.

The laboratory findings were mean leukocyte count was 9,951 $\pm 2,250 / \mathrm{mm}^{3}$, mean ESR was $54.04 \pm 28.5 \mathrm{~mm} / \mathrm{hr}$, mean CRP was $24.8 \pm 18.6 \mathrm{~g} / \mathrm{dL}$. The MAT antibody titers were found to be $1: 640$ for 9 patients, and 1:1,280 for others.

All patients received medical treatment. Nine patients received initial medical therapy (intramuscular streptomycin for 14 days) but did not respond. Six of the 9 patients required surgical drainage, 2 patients required total lymph node excision, and fine needle aspiration biopsy (FNA) was performed on 1 patient. Following surgery, ciprofloxacin was given orally for 3 weeks in all cases.

Five patients received initial treatment with oral ciprofloxacin; the duration of the ciprofloxacin treatment was 4-7 weeks. Two of 5 patients underwent surgical drainage, and total lymph node excision was performed on 1 patient due to the lack of significant reduction in lymph node size during medical treatment.

First-line gentamicin was given to 4 patients. After 1 patient had no reduction in lymph node size during intravenous treatment for 10 days, streptomycin was started and given for 14 days. One patient required surgical drainage, and 2 patients underwent total lymph node excision. Oral ciprofloxacin was given for 3 weeks after surgery in these 3 cases.

Initially, 1 patient was given oral doxycycline for 14 days. Because the patient had no reduction in the size of the lymph node, FNA was performed, and oral ciprofloxacin was given for 4 weeks.

Totally, surgical incision and drainage were performed for 9 patients, 2 patients underwent FNA, and 5 patients underwent total mass excision (Table 2). Surgical procedures (performed by an otolaryngologist) were used both for the diagnosis and for treatment. The pathologic examinations revealed chronic granulomatous inflammatory events in the biopsied cases.

\section{Discussion}

Tularemia may develop granulomatous and suppurative lesions, especially in the affected regional lymph nodes and in various organs. ${ }^{10,11)}$ Before the appearance of the affected lymph node, early symptoms are very nonspecific, such as sore throat, fever, and malaise. Because of this clinical uncertainty, before being diagnosed, a large portion of patients receive various medical treatments. This condition increases both morbidity and costs. Delay in diagnosis and 
Table 2. Relationship between medical treatment and surgical intervention

\begin{tabular}{lcccccc}
\hline First-line treatment & Number & Treatment failure & Surgical drainage & FNAB & Total mass excision & Second-line treatment \\
\hline Streptomycin (2 wk) & 9 & 9 & 6 & 1 & 2 & Ciprofloxacin (3 wk) \\
Ciprofloxacin (4-7 wk) & 5 & 3 & 2 & - & - \\
Gentamicin (10 days) & 4 & 3 & 1 & - & - & Streptomycin (2 wk - no surgery) \\
Ciprofloxacin (3 wk) & - & - & - & 1 & - & Ciprofloxacin (4 wk) \\
Doxycycline (2 wk) & 1 & 1 & 9 & 2 & 5 & - \\
Total & 19 & 16 & & & - \\
\hline
\end{tabular}

FNAB, fine needle aspiration biopsy.

effective treatment can lead to growth of the lymph nodes, and the formation of abscesses. In particular, if the period between the start of symptoms and the onset of effective treatment is longer than 3 weeks, it may because negative clinical outcomes. ${ }^{12)}$ In our patients, before the diagnosis, the duration of symptoms was $32.15 \pm 17.8$ days. All of our study population who were referred to our tertiary care university hospital had no response to the various medical treatments they had previously received. Therefore, the lymph node suppuration and surgical intervention rates were high among our cases. Prior to a definitive diagnosis, most of the empirical treatment received are $\beta$-lactam antimicrobial drugs, whereas most $\beta$-lactams have no bacteriostatic activity against $F$. tularensis in vitro. ${ }^{6}$

Antibiotic treatment of tularemia includes several drugs, including aminoglycosides (e.g., streptomycin and gentamicin), tetracyclines (e.g., doxycycline), and fluoroquinolones (e.g., ciprofloxacin). ${ }^{13,14)}$ Because of the potential toxicity of first-line drugs, high rates of relapse, and intractable suppurate forms of tularemia, this implies that therapeutic alternatives are not enough for the effective treatment of tularemia, especially in children. ${ }^{11)}$

Among treatment options, aminoglycosides (e.g., streptomycin and gentamicin) are the first preferred antibiotics; in vitro studies have shown bactericidal activity against $F$. tularensis, and that they have better outcomes and lower relapse rates. Their usage is limited, however, based on 2 factors: they can only be used parenterally, and they may cause toxicity (especially ototoxicity and nephrotoxicity). ${ }^{15)} \mathrm{A}$ few authors have said that gentamicin treatment could fail in pediatric patients with oropharyngeal tularemia. Kaya et al. ${ }^{16)}$ evaluated eleven pediatric patients with oropharyngeal tularemia, 4 of whom had received gentamicin for first-line therapy and had undergone surgical drainage due to suppuration development; the authors reported treatment success when the therapy was switched to streptomycin. In our study, we saw treatment failure in 4 cases who had been given gentamicin in initial therapy; 3 of these 4 patients underwent surgical procedure, and one patient's treatment was switched to streptomycin. Enderlin et al. ${ }^{15)}$ reported in their metaanalysis that the rate of cure for streptomycin was 97\%, with no relapses.

A few authors have suggested the use of fluoroquinolones as first-line drugs in recent years. They have been used especially in patients with mild to moderate clinical tularemia. Studies have reported that fluoroquinolones (e.g., ciprofloxacin and levofloxacin) have advantages such as excellent bioavailability and activity, accessibility of oral formulations, and lower toxicities. Ciprofloxacin cannot be usedin children aged $<12$ years, however, due to its adverse effects on cartilage. However, some clinical experience shows that it is safe in children. ${ }^{2,17)}$ In our study, patients given ciprofloxacin showed longer treatment durations, of up to 7 weeks.

Other potential alternative drugs include tetracyclines (especially doxycycline). Two major drawbacks have been reported for tetracyclines, however the necessity for long-term usage, and higher relapse rates when compared to fluoroquinolones. In addition to these factors that limit the use of tetracyclines, classically they are contraindicated in children due to the risk of teeth discoloration. ${ }^{14,15}$ The patient given tetracycline in our study had no reduction in lymph node, and underwent FNA. Recovery was seen only after 4 weeks of ciprofloxacin treatment.

In Turkey, the majority of patients with tularemia have cervical lymph nodes when consulted by a physician. The antibiotic therapy initiation time is very important, since delayed lymph node may become suppurated. In addition, without any treatment, lymphadenopathy and suppuration may last for several months. In case of the persistence of cervical lymph nodes despite antibiotic treatment, or developing abscess formation, patients should be consulted to an otolaryngologist. ${ }^{11)}$ Following an effective medical treatment, an increase in size, or the appearance of new, enlarged, or suppurated lymph nodes generally should be considered as treatment failure. A study from Spain reported that the rate of treatment failure was 22.5 percent. ${ }^{7)}$ Surgical procedures were performed for 16 patients in our study. It is commonly known that if patients are unresponsive to medical treatment, biopsy may be needed to eliminate malignity and other differential diagnoses. In our study, 5 patients underwent total mass excision; FNA was also performed for 2 patients, and the pathologic examinations revealed chronic granulomatous inflammatory events. But these results may be confused in some countries such as Turkey where tuberculosis is common. In this case, the diagnosis must be supported by detailed anamnesis and serological tests.

Tezer et al. ${ }^{8)}$ described that some of the factors that lead to com- 
mon treatment failures include sex (specifically, being female), treatment delay $>16$ days, and doxycycline use. $0 z$ et al. ${ }^{12)}$ did not find an association between treatment failure and treatment modalities, but they did find that delays in treatment caused the formation of abscesses in the lymph nodes.

The most important limitations of the present study were being a single-hospital study and having a small sample size. For that reason, we can assert that our results do not represent the general pediatric patients with tularemia.

In conclusion, tularemia should be considered (especially in endemic regions) when a patient develops fever, pharyngitis-tonsillitis, or cervical lymphadenopathy, and shows no response to $\beta$-lactam antimicrobial drugs. None of our study population who were referred to our tertiary care hospital responded to various previously received medical treatments. Antimicrobial initiation time is crucial in these cases, since lymph nodes may be suppurated in case of any delay in proper treatment. First-line therapies have several drawbacks in the effective treatment of tularemia, such as potential toxicity, high relapse rates, persistent suppurative forms, and inadequate treatment alternatives. In our experience, delay and ineffectiveness in early treatment, as well as the development of suppurated lymph nodes, are the major causes of treatment failure in tularemia. We should keep in mind that surgical procedures might be necessary when there is no response to antimicrobial treatment.

\section{Conflicts of interest}

No potential conflict of interest relevant to this article was reported.

\section{References}

1. Lamps LW, Havens JM, Sjostedt A, Page DL, Scott MA. Histologic and molecular diagnosis of tularemia: a potential bioterrorism agent endemic to North America. Mod Pathol 2004;17:489-95.

2. Kiliç S, Celebi B, Acar B, Ataş M. In vitro susceptibility of isolates of Francisella tularensis from Turkey. Scand J Infect Dis 2013;45:33741.
3. Ulu-Kilic A, Gulen G, Sezen F, Kilic S, Sencan I. Tularemia in central Anatolia. Infection 2013;41:391-9.

4. Gozel MG, Engin A, Altuntas EE, Salk İ, Kaya A, Celik C, et al. Evaluation of clinical and laboratory findings of pediatric and adult patients with oropharyngeal tularemia in Turkey: a combination of surgical drainage and antibiotic therapy increases treatment success. Jpn J Infect Dis 2014;67:295-9.

5. Ellis J, Oyston PC, Green M, Titball RW. Tularemia. Clin Microbiol Rev 2002;15:631-46.

6. Tärnvik A, Chu MC. New approaches to diagnosis and therapy of tularemia. Ann N Y Acad Sci 2007;1105:378-404.

7. Pérez-Castrillón JL, Bachiller-Luque P, Martín-Luquero M, MenaMartín FJ, Herreros V. Tularemia epidemic in northwestern Spain: clinical description and therapeutic response. Clin Infect Dis 2001; 33:573-6.

8. Tezer H, Ozkaya-Parlakay A, Aykan H, Erkocoglu M, Gülhan B, Demir A, et al. Tularemia in children, Turkey, September 2009-November 2012. Emerg Infect Dis 2015;21:1-7.

9. Kosker M, Sener D, Kilic O, Akil F, Yilmaz M, Ozturk O, et al. A case of oculoglandular tularemia resistant to medical treatment. Scand J Infect Dis 2013;45:725-7.

10. Park CH, Nakanishi A, Hatai H, Kojima D, Oyamada T, Sato H, et al. Pathological and microbiological studies of Japanese Hare (Lepus brachyurus angustidens) naturally infected with Francisella tularensis subsp. holarctica. J Vet Med Sci 2009;71:1629-35.

11. Kızıl Y, Aydil U, Cebeci S, Güzeldir OT, Inal E, Bayazıt Y. Characteristics and management of intractable neck involvement in tularemia: report of 19 patients. Eur Arch Otorhinolaryngol 2012;269:1285-90.

12. Oz F, Eksioglu A, Tanır G, Bayhan G, Metin Ö, Teke TA. Evaluation of clinical and sonographic features in 55 children with tularemia. Vector Borne Zoonotic Dis 2014;14:571-5.

13. Penn RL. Francisella tularensis (tularemia). In: Mandell GL, Bennett JE, Dolin R, editors. Mandell, Douglas and Bennett's principles and practice of infectious diseases, 7th ed. Philadelphia (PA): ElsevierChurchill Livingstone, 2010:2927-37.

14. Boisset S, Caspar Y, Sutera V, Maurin M. New therapeutic approaches for treatment of tularaemia: a review. Front Cell Infect Microbiol 2014;4:40.

15. Enderlin G, Morales L, Jacobs RF, Cross JT. Streptomycin and alternative agents for the treatment of tularemia: review of the literature. Clin Infect Dis 1994;19:42-7.

16. Kaya A, Uysal IÖ, Güven AS, Engin A, Gültürk A, İçağasıŏlu FD, et al. Treatment failure of gentamicin in pediatric patients with oropharyngeal tularemia. Med Sci Monit 2011;17:CR376-80.

17. Kreizinger Z, Makrai L, Helyes G, Magyar T, Erdélyi K, Gyuranecz M. Antimicrobial susceptibility of Francisella tularensis subsp. holarctica strains from Hungary, Central Europe. J Antimicrob Chemother 2013; 68:370-3. 